\title{
ON A FUNDAMENTAL RELATION IN ABSTRACT GEOMETRY.
}

BY MR. A. R. SCHWEITZER.

(Read before the American Mathematical Society, September 3, 1906.)

1. Is his Abstrakte Geometrie, page 10, Professor Vahlen seeks to define planar order with the aid of two relations, each of which is between a point and a set of linearly ordered points, such that if $b c$ and $b_{1} c_{1}$ are any two pairs of distinct points of the set, $b$ precedes (follows) $c$ and $b_{1}$ precedes (follows) $c_{1}$ simultaneously. The linear order referred to is established by means of the undefined relation due to Vailati which may be expressed concretely by "precede." Aside from the fact that Vahlen employs a superfluity of undefined symbols, there is a noticeable lack of precision in his presentation.* Recent investigations of the writer have emphasized the importance, in abstract geometry, of a relation used implicitly by Vahlen, which, however, seems to have escaped him entirely. The object of the present note is briefly to point this out.

2. The relation referred to above is $a R(b c)$, which may be considered an abstract statement for the following: "If a person swims from $b$ to $c$ the point $a$ is at his right." In the relation $a R(b c),(b c)$ is an ordered dyad. That is, if $b \neq c$, then the dyads $(b c)$ and $(c b)$ are distinct, and if the points $b c$ are not the points $b_{1} c_{1}$, then the dyads $(b c)$ and $\left(b_{1} c_{1}\right)$ are also distinct. Thus if $(b c)$ and $\left(b_{1} c_{1}\right)$ are identical, ordered dyads, then $b=b_{1}$ and $c=c_{1}$. The existence of an ordered dyad, it will be observed, does not properly involve any geometric assumptions, except the existence of points.

3. We can now construct planar axioms and definitions which will clarify the Vahlen text.

\section{Axioms.}

(1) There is at least one point.

(2) If there exists a point, there exist the points $a_{0}, b_{0}, c_{0}$ such that $a_{0} R\left(b_{0} c_{0}\right)$ or $a_{0} R\left(c_{0} b_{0}\right)$.

(3) If $a R(b c)$, then $a \neq b, c$.

* Cf. the review by Professor Veblen, Bulletin, July, 1906, p. 505. 
(4) If $a R(b c)$ and $b \neq c$, then $a \bar{R}(c b) .^{*}$

(5) If $a R(b c)$ and $x \neq a$, then $x R(b c)$ or $x R(c a)$ or $x R(a b)$.

\section{Definitions.}

(1) The point $x$ is collinear with $(a b)$ if $a \neq b, x \bar{R}(a b)$, and $x \bar{R}(b a)$.

(2) The point $x$ is in the interior of $(a b)$ if

there is a point $c^{\prime}$ such that $c^{\prime} R(a b)$,

$x \bar{R}(a b), x \bar{R}(b a)$,

for any point $c$ such that $c R(a b)$ and $x R(b c)$ or $x R(c a)$ follows $x R(b c), x R(c a)$.

(3) The point $x$ is in the plane (abc) if $a R(b c)$ and either $x R(b c)$ or $x R(c a)$ or $x R(a b)$.

(4) The point $x$ is in the interior of $(a b c)$ if $x R(b c), x R(c a)$, and $x R(a b)$.

It will be noticed that it is not necessary to introduce an additional relation $a L(b c)$ as Vahlen does. This relation is expressed by $a R(c b)$. The definitions (1)-(3) are not given by Vahlen.

4. As a consequence of the preceding axioms we prove the following (Cf. Vahlen, Axiom, § 21, page 10).

Theorem 1. If $c R(a b)$, then $a R(b c)$ and $b R(c a)$.

Since $c R(a b)$, by axiom (3), $c \neq a, b$. Hence by axiom (5), $a R(a b)$ or $a R(b c)$ or $a R(c a)$. Therefore by (3), $a R(b c)$. Similarly, $b R(c a)$.

Theorem 2. If $x R(a b)$ and $x R(b c)$ and $x R(c a)$, then $c R(a b)$.

We may assume $c \neq x$. Then by axiom (5), $c R(a b)$ or $c R(x a)$ or $c R(b x)$. By theorem 1, if $c R(x a)$ or $c R(b x)$, then $x R(a c)$ or $x R(c b)$ respectively. Hence by axioms (3), (4), and the hypothesis, $c R(a b)$ is the only possibility. The theorem therefore follows.

5. It would be easy to construct further axioms so that the preceding definitions are effective. For this purpose it will be most convenient to refer to the writer's planar descriptive system. $\uparrow$ There results, then, that by using two undefined symbols, point and the (planar) relation $a R(b c)$, it is possible to construct a set of axioms for the plane without introducing an undefined linear relation as Vahlen has done. However, the relation $a R(b c)$ or, as we will now write it, $a R_{2}(b c)$ may be considered an extension, dimensionally, of the undefined relation

* The rule over the $R$ is a symbol of negation.

$\dagger$ Cf. Bulletin, June, 1906, p. 438 . For $n$-ad read $(n+1)$-ad. 
of Vailati, which we will write $a R_{1} b$. More generally, it is possible to construct a set of $n$-dimensional axioms $(n=1,2,3, \cdots)$ by means of the two undefined symbols point and the $n$-dimensional relation $a R_{n}\left(b_{1} b_{2} \cdots b_{n}\right)$, where the $n$-ad is ordered.

ChICAgo,

July, 1906.

\section{ON THE ORDERLY LISTING OF SUB- STITUTIONS.}

BY PROFESSOR D. N. LEHMER.

(Read before the San Francisco Section of the American Mathematical Society, February 24, 1906.)

1. In a substitution such as $\left(\begin{array}{c}12345 \\ 34251\end{array}\right)$ we shall speak of 34251 as the permutation belonging to the substitution. The following list of the permutations belonging to the substitutions on four letters exhibits what seems to be the most orderly arrangement :

$$
\begin{array}{rl}
1=1234=\mathrm{I} & 13=3124=(132) \\
2=1243=(34) & 14=3142=(1342) \\
3=1324=(23) & 15=3214=(13) \\
4=1342=(234) & 16=3241=(134) \\
5=1423=(243) & 17=3412=(13)(24) \\
6=1432=(24) & 18=3421=(1324) \\
7=2134=(12) & 19=4123=(1432) \\
8=2143=(12)(34) & 20=4132=(142) \\
9=2314=(123) & 21=4213=(143) \\
10=2341=(1234) & 22=4231=(14) \\
11=2413=(1243) & 23=4312=(1423) \\
12=2431=(124) & 24=4321=(14)(23)
\end{array}
$$

2. It is seen that the numbers of four digits expressed by the different permutations are in ascending order of magnitude. This order would be reversed by the interchange of 1 with 4 and 2 with 3.

3. It is easily possible to compute the rank of a given permutation or to write down a permutation of given rank. Thus the rank of the permutation 341562 is 240 plus the rank 